\title{
Terpenes from Artemisia herba-alba
}

\author{
Abou El-Hamd H. Mohamed*, Abeer M. Esmail, and Adila M. El-Saade \\ Department of Chemistry, Aswan-Faculty of Science, Aswan University, \\ Aswan, Egypt. Fax: +020973480450. E-mail: abouhassan68@yahoo.com \\ * Author for correspondence and reprint requests \\ Z. Naturforsch. 68c, 343-346 (2013); received December 22, 2012/May 5, 2013 \\ Chromatographic investigation of the methylene chloride/methanol extract of the aerial \\ parts of Artemisia herba-alba afforded a new monoterpene dimer, in addition to a known \\ monoterpene and three known sesquiterpene lactones. The structures of the compounds \\ were determined by comprehensive NMR analyses, including DEPT, COSY, HMQC, \\ HMBC, and HRMS.
}

Key words: Artemisia herba-alba, Sesquiterpenes, Monoterpene

\section{Introduction}

The large genus Artemisia, family Asteraceae in the tribe Anthemideae, has been the subject of numerous chemical and biological studies (Saadali et al., 2001). Artemisia species, widespread throughout the world, are important medicinal plants, which have been used for the treatment of diseases such as malaria, hepatitis, cancer, tracheitis, pharyngitis, pneumonia, inflammation, and infections by fungi, bacteria, and viruses (Kim et al., 2002; Zheng et al., 1996). Several species of Artemisia are important in folk medicine, thus, $A$. herba-alba has been used as an antihelminthic by local populations in Morocco (Boriky et al., 1996), A. mongolica has been proven to cure inflammations and colds in Northwest China (Hu et al., 1996), A. pontica is used as a sedative and appetizer in Bulgarian folk medicine (Todorova et al., 1996). Of approximately 200 species growing in China, more than 50 have been used in traditional Chinese medicinal practice, for the treatment of gynaecopathy, amenorrhea, bruise, and rheumatic disease (Tan et al., 1999; Kwak et al., 1997). Furthermore, A. vulgaris is used to flavour tea and rice dishes in Asia and as a culinary herb for poultry and pork in western cultures. In oriental medicine, it has been employed as an analgesic agent, in conjunction with acupuncture therapy, and has been implemented in the treatment of painful menstruation and in the induction of labour or miscarriage (Lee et al., 1998). From extensive studies, the genus Artemisia has been found to be a rich source of biologically active compounds such as monoterpenes, ses- quiterpenes, triterpenes, and flavones (Kim et al., 2002; Tan et al., 1999; Tang et al., 2000; Mohamed et al., 2010). This paper describes the isolation, identification, and structure elucidation of a new monoterpene dimer, $\mathbf{1}$, two germacranolides, 2 and 3 (Marco 1989; Marco et al., 1994; Pathak and Khanna, 1987), an eudesmanolide, 4 (Ahmed et al., 1990), and a monoterpene, 5 (Marco et al., 1991), from the aerial parts of $A$. herba-alba.

\section{Results and Discussion}

Repetitive chromatographic steps in the fractionation of the methylene chloride/methanol (1:1) extract of the air-dried aerial parts of $A$. herba-alba afforded the new compound $\mathbf{1}$, in addition to the known sesquiterpene lactones $\mathbf{2}, \mathbf{3}$, and 4 and the monoterpene 5. Compound 1 was obtained as yellowish oil and showed a molecular ion peak $[\mathrm{M}]^{+}$at $m / z 360$, in accord with the molecular formula $\mathrm{C}_{21} \mathrm{H}_{28} \mathrm{O}_{5}$, in the EI mass spectrum. Fragment ions at $m / z \quad 342$ and 327 were due to the elimination of a water molecule and a methyl group, respectively. An interesting peak appearing at $\mathrm{m} / \mathrm{z} 300(12 \%)$ was due to the elimination of a $\mathrm{CH}_{3} \mathrm{COOH}$ molecule from the $[\mathrm{M}]^{+}$ion. The strong peak at $\mathrm{m} / z 135\left[\mathrm{C}_{9} \mathrm{H}_{11} \mathrm{O}\right]^{+}(33 \%)$ resulted from the elimination of $\mathrm{C}_{10} \mathrm{H}_{13} \mathrm{O}_{2}$ from the $[\mathrm{M}-$ $\left.\mathrm{CH}_{3} \mathrm{COOH}\right]^{+}$ion. This ion could be formed by cleavage of the $\mathrm{C} 9-\mathrm{C} 10$ bond. The fragment ion at $\mathrm{m} / z 105$ (40\%) might be produced by elimination of the two methyl groups from the ion 135, while the fragment ion at $\mathrm{m} / \mathrm{z} 91$ could be produced by elimination of a $\mathrm{CH}_{2}$ group from the ion 105 . Exact mass determination of the ion at $\mathrm{m} / \mathrm{z}, 360$ 
<smiles>[Y]C1([2H])C=C(CC2C=C(C)C(C)(C(=O)OC)CC2=O)C2CC(=O)O[C]21</smiles><smiles>CC1=CC2CCC[C@](C)(CC1)C(=O)O2</smiles>

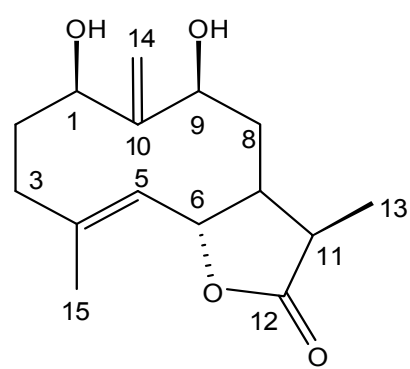

2<smiles>C=C1CCC(O)C2(C)C(=O)OC1C1CC(C)=C(C)C1=CC2O</smiles>

4<smiles>CC1=CC(C)(C)C(C(=O)O)CC1=O</smiles>

5

Fig. 1. Chemical structures of compounds 1-5 isolated from Artemisia herba-alba.

established the elemental composition $\mathrm{C}_{21} \mathrm{H}_{28} \mathrm{O}_{5}$ (experimental 360.1937, calcd. 360.1902).

The ${ }^{1} \mathrm{H}$ NMR spectrum showed seven singlet signals at $\delta_{\mathrm{H}} 6.30 \mathrm{ppm}, 1.10 \mathrm{ppm}, 1.28 \mathrm{ppm}$, $5.08 \mathrm{ppm}, 1.22 \mathrm{ppm}, 1.12 \mathrm{ppm}$, and $3.70 \mathrm{ppm}$ assigned to $\mathrm{H}-2$ ', $\mathrm{H}-7^{\prime}, \mathrm{H}-8^{\prime}, \mathrm{H}-5, \mathrm{H}-11, \mathrm{H}-12$, and the methyl ester, respectively. Furthermore, it revealed the presence of a doublet of doublets at $\delta_{\mathrm{H}} 2.88 \mathrm{ppm}\left(J=11.9,4.3 \mathrm{~Hz}, \mathrm{H}-4^{\prime}\right)$, correlated with a doublet of doublets at $\delta_{\mathrm{H}} 2.52 \mathrm{ppm}(\mathrm{dd}, J=$ $4.3,17.0 \mathrm{~Hz}, \mathrm{H}-5$ 'a) and $2.69 \mathrm{ppm}(\mathrm{dd}, J=11.9$, $\left.17.0 \mathrm{~Hz}, \mathrm{H}-5^{\prime} \mathrm{b}\right)$ in the ${ }^{1} \mathrm{H}-{ }^{1} \mathrm{H}-\mathrm{COSY}$ spectrum. It also showed a doublet signal at $\delta_{\mathrm{H}} 4.56 \mathrm{ppm}(\mathrm{d}$, $J=4.55 \mathrm{~Hz}, \mathrm{H}-7$ ), coupled with a carbon atom at $\delta_{\mathrm{C}} 77.74 \mathrm{ppm}(\mathrm{C}-7)$ in the HMQC spectrum. The latter proton showed a correlation with a multiplet signal at $\delta_{\mathrm{H}} 2.33 \mathrm{ppm}(\mathrm{m}, \mathrm{H}-8)$ in the ${ }^{1} \mathrm{H}-{ }^{1} \mathrm{H}$-COSY spectrum. Moreover, it showed a multiplet signal at $\delta_{\mathrm{H}} 2.14 \mathrm{ppm}$ integrated for three protons (H-3a, H-9a, H-10a), and it showed a clear correlation in the ${ }^{1} \mathrm{H}-{ }^{1} \mathrm{H}-\mathrm{COSY}$ spectrum with a multiplet signal at $\delta_{\mathrm{H}} 2.38 \mathrm{ppm}$ integrated for three protons (H-3b, H-9b, H-10b). The ${ }^{13} \mathrm{C}$ NMR and DEPT experiments of $\mathbf{1}$ displayed twenty one carbon signals: three carbonyl carbon atoms at $\delta_{\mathrm{C}} 177.0 \mathrm{ppm}(\mathrm{C}-2), 197.27 \mathrm{ppm}\left(\mathrm{C}-6^{\prime}\right)$, and $172.62 \mathrm{ppm}$ (C-9'), five methyl carbon atoms at $\delta_{\mathrm{C}} 28.27 \mathrm{ppm}$ (C-11), $27.40 \mathrm{ppm}$ (C-12), $22.91 \mathrm{ppm}\left(\mathrm{C}^{-} 7^{\prime}\right), 28.93 \mathrm{ppm}\left(\mathrm{C}-8^{\prime}\right)$, and $51.69 \mathrm{ppm}$ $(\mathrm{COOMe})$, four methylene carbon atoms at $\delta_{\mathrm{C}} 34.08 \mathrm{ppm}(\mathrm{C}-3), 36.78 \mathrm{ppm}\left(\mathrm{C}-5^{\prime}\right), 26.98 \mathrm{ppm}$ (C-9), and $33.76 \mathrm{ppm}$ (C-10), five methine carbon atoms at $\delta_{\mathrm{C}} 154.37 \mathrm{ppm}\left(\mathrm{C}-2^{\prime}\right), 49.40 \mathrm{ppm}$ (C-4'), 133.49 ppm (C-5), 77.74 ppm (C-7), and $48.96 \mathrm{ppm}$ (C-8), and four quaternary carbon atoms at $\delta_{\mathrm{C}} 136.0 \mathrm{ppm}\left(\mathrm{C}-1^{\prime}\right), 35.15 \mathrm{ppm}\left(\mathrm{C}-3^{\prime}\right)$, $134.75 \mathrm{ppm}$ (C-4), and $35.46 \mathrm{ppm}$ (C-6). Confirmation of the structure of compound $\mathbf{1}$ was given by the HMBC analysis; the most important correlations were observed between: $\mathrm{H}-2^{\prime}\left(\delta_{\mathrm{H}} 6.30 \mathrm{ppm}\right.$, s) and C-4' $\left(\delta_{\mathrm{C}} 49.40 \mathrm{ppm}\right), \mathrm{C}-6^{\prime}\left(\delta_{\mathrm{C}} 197.27 \mathrm{ppm}\right)$, C-10 $\left(\delta_{\mathrm{C}} 33.76 \mathrm{ppm}\right) ; \mathrm{H}-4{ }^{\prime}\left(\delta_{\mathrm{H}} 2.88 \mathrm{ppm}, \mathrm{dd}\right)$ 
and C-3' $\left(\delta_{\mathrm{C}} 35.15 \mathrm{ppm}\right), \mathrm{C}-5^{\prime}\left(\delta_{\mathrm{C}} 36.78 \mathrm{ppm}\right)$,

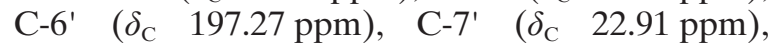
C-8' $\left(\delta_{\mathrm{C}} 28.93 \mathrm{ppm}\right), \quad \mathrm{C}-9^{\prime} \quad\left(\delta_{\mathrm{C}} 172.62 \mathrm{ppm}\right)$; $\mathrm{H}-5^{\prime} \quad\left(\delta_{\mathrm{H}} 2.52 \mathrm{ppm}, \mathrm{dd} ; 2.69 \mathrm{ppm}, \mathrm{dd}\right)$ and C-3' $\left(\delta_{\mathrm{C}} 35.15 \mathrm{ppm}\right), \mathrm{C}-4^{\prime}\left(\delta_{\mathrm{C}} 49.40 \mathrm{ppm}\right), \mathrm{C}-6{ }^{\prime}$ $\left(\delta_{\mathrm{C}} 197.27 \mathrm{ppm}\right) ; \mathrm{H}-3\left(\delta_{\mathrm{H}} 2.14 \mathrm{ppm}, \mathrm{m} ; 2.38 \mathrm{ppm}\right.$, $\mathrm{m})$ and $\mathrm{C}-2\left(\delta_{\mathrm{C}} 177.0 \mathrm{ppm}\right)$; H-5 $\left(\delta_{\mathrm{H}} 5.08 \mathrm{ppm}\right.$, s) and C-6 $\left(\delta_{\mathrm{C}} 35.46 \mathrm{ppm}\right), \mathrm{C}-7\left(\delta_{\mathrm{C}} 77.74 \mathrm{ppm}\right)$, C-8 $\left(\delta_{\mathrm{C}} 48.96 \mathrm{ppm}\right), \mathrm{C}-9 \quad\left(\delta_{\mathrm{C}} 26.98 \mathrm{ppm}\right) ; \mathrm{H}-7$ $\left(\delta_{\mathrm{H}} 4.56 \mathrm{ppm}, \mathrm{d}\right)$ and $\mathrm{C}-2\left(\delta_{\mathrm{C}} 177.0 \mathrm{ppm}\right), \mathrm{C}-4$ $\left(\delta_{\mathrm{C}} 134.75 \mathrm{ppm}\right), \mathrm{C}-8\left(\delta_{\mathrm{C}} 48.96 \mathrm{ppm}\right) ; \mathrm{H}-8\left(\delta_{\mathrm{H}} 2.33\right.$ ppm, m) and C-6 $\left(\delta_{\mathrm{C}} 35.46 \mathrm{ppm}\right) ; \mathrm{H}-9\left(\delta_{\mathrm{H}} 2.14 \mathrm{ppm}\right.$, $\mathrm{m} ; 2.38 \mathrm{ppm}, \mathrm{m}), \mathrm{H}-10\left(\delta_{\mathrm{H}} 2.14 \mathrm{ppm}, \mathrm{m} ; 2.38 \mathrm{ppm}\right.$, $\mathrm{m})$ and $\mathrm{C}-4\left(\delta_{\mathrm{C}} 134.75 \mathrm{ppm}\right), \mathrm{C}-1^{\prime}\left(\delta_{\mathrm{C}} 136.0 \mathrm{ppm}\right)$; $\mathrm{H}-11\left(\delta_{\mathrm{H}} 1.22 \mathrm{ppm}\right), \mathrm{H}-12\left(\delta_{\mathrm{H}} 1.12 \mathrm{ppm}\right)$ and C-5 $\left(\delta_{\mathrm{C}} 133.49 \mathrm{ppm}\right) ; \mathrm{H}-7^{\prime}\left(\delta_{\mathrm{H}} 1.10 \mathrm{ppm}\right), \mathrm{H}-8^{\prime}$ $\left(\delta_{\mathrm{H}} 1.28 \mathrm{ppm}\right)$, and $\mathrm{C}-2^{\prime}\left(\delta_{\mathrm{C}} 154.37 \mathrm{ppm}\right), \mathrm{C}-3^{\prime}$ $\left(\delta_{\mathrm{C}} 35.15 \mathrm{ppm}\right), \mathrm{C}-4^{\prime}\left(\delta_{\mathrm{C}} 49.40 \mathrm{ppm}\right)$. On the basis of these results, compound $\mathbf{1}$ was identified as a new monoterpene dimer, which was given the name herbalbin, a new natural product (Fig. 1).

\section{Experimental}

\section{General}

${ }^{1} \mathrm{H} \mathrm{NMR}\left(600 \mathrm{MHz}, \mathrm{CDCl}_{3}\right),{ }^{13} \mathrm{C} \mathrm{NMR}(125 \mathrm{MHz}$, $\mathrm{CDCl}_{3}$ ), and the $2 \mathrm{D}$ spectra were recorded on a JEOL (Hiroshima, Japan) 500-MHz Lambda spectrometer, with tetramethylsilane (TMS) as an internal standard. EI mass spectra were recorded on a JEOL SX102A mass spectrometer.

\section{Plant material}

The aerial parts of $A$. herba-alba Asso were collected during the flowering stage at Tebessa, Eastern Algeria. A voucher specimen has been deposited at the Department of Botany, El-Minia University, El-Minia, Egypt.

\section{Extraction and isolation}

Air-dried aerial plant material (400 g) was ground and extracted with $\mathrm{CH}_{2} \mathrm{Cl}_{2} / \mathrm{MeOH}$ (1:1, $\mathrm{v} / \mathrm{v})$ at room temperature for $24-48 \mathrm{~h}$. The extract was concentrated in vacuo to obtain a residue of $60 \mathrm{~g}$. The residue was pre-fractionated by column chromatography (CC) on a silica gel column $(6 \mathrm{~cm}$ x $120 \mathrm{~cm}$ ) eluted with $n$-hexane $(2 \mathrm{~L})$ followed by a gradient of $n$-hexane $/ \mathrm{CH}_{2} \mathrm{Cl}_{2}$ up to $100 \% \mathrm{CH}_{2} \mathrm{Cl}_{2}$ and $\mathrm{CH}_{2} \mathrm{Cl}_{2} / \mathrm{MeOH}$ up to $15 \% \mathrm{MeOH}(2 \mathrm{~L}$ each of the solvent mixtures). The $n$-hexane/ $\mathrm{CH}_{2} \mathrm{Cl}_{2}(1: 1$, $\mathrm{v} / \mathrm{v}, 6 \mathrm{~g})$ fraction was subjected to silica gel $\mathrm{CC}$ $(2 \mathrm{~cm} \times 60 \mathrm{~cm})$, eluted with $n$-hexane $/ \mathrm{CH}_{2} \mathrm{Cl}_{2}$ with increasing polarity up to $100 \% \mathrm{CH}_{2} \mathrm{Cl}_{2}$, to give pure compound 2 (10 mg). The $\mathrm{CH}_{2} \mathrm{Cl}_{2}(100 \%)$ fraction was chromatographed on a Sephadex LH-20 column eluted with $n$-hexane/ $\mathrm{CH}_{2} \mathrm{Cl}_{2} / \mathrm{MeOH}$ (7:4:0.5, $\mathrm{v} / \mathrm{v} / \mathrm{v})$ to give compounds $3(10 \mathrm{mg}), \mathbf{4}(15 \mathrm{mg})$, and 5 (11 mg). The $\mathrm{CH}_{2} \mathrm{Cl}_{2} / \mathrm{MeOH}(95: 5, \mathrm{v} / \mathrm{v})$ fraction was chromatographed on a Sephadex LH-20 column eluted with $n$-hexane/ $\mathrm{CH}_{2} \mathrm{Cl}_{2} / \mathrm{MeOH}$ (7:4:0.5) to give compound $\mathbf{1}(6 \mathrm{mg})$.

Herbalbin (1): Yellowish oil. - IR (KBr): $v=$ 1716 (broad) $(\mathrm{C}=\mathrm{O}), 1623 \mathrm{~cm}^{-1}(\mathrm{C}=\mathrm{C}) .-{ }^{1} \mathrm{H}$ NMR [the ${ }^{1} \mathrm{H}$ assignments were achieved by ${ }^{1} \mathrm{H}-{ }^{1} \mathrm{H}$ correlation spectroscopy (COSY)]: $\delta=6.30\left(\mathrm{~s}, \mathrm{H}-2^{\prime}\right)$, $2.88\left(\mathrm{dd}, J=11.9,4.3 \mathrm{~Hz}, \mathrm{H}-4^{\prime}\right), 2.52(\mathrm{dd}, J=4.3$, 17.0 Hz, H-5'a), 2.69 (dd, $J=11.9,17.0 \mathrm{~Hz}, \mathrm{H}-5$ 'b), 1.10 (s, H-7'), 1.28 (s, H-8'), 2.14 (m, H-3a), 2.38 (m, H-3b), 5.08 (s, H-5), 4.56 (d, $J=4.55 \mathrm{~Hz}, \mathrm{H}-7$ ), 2.33 (m, H-8), 2.14 (m, H-9a), 2.38 (m, H-9b), 2.14 (m, H-10a), 2.38 (m, H-10b), 1.22 (s, H-11), 1.12 (s, H-12), 3.70 (s, methyl of acetate). $-{ }^{13} \mathrm{C}$ NMR (the ${ }^{13} \mathrm{C}$ assignments were achieved by HMQC and HMBC): $\delta=136.0$ (s, C-1'), 154.37 (d, C-2'), 35.15 (s, C-3'), 49.40 (d, C-4'), 36.78 (t, C-5'), 197.27 (s, C-6'), 22.91 (q, C-7'), 28.93 (q, C-8'), 172.62 (s, C-9'), 177.0 (s, C-2), 34.08 (t, C-3), 134.75 (s, C-4), 133.49 (d, C-5), 35.46 (s, C-6), 77.74 (d, C-7), 48.96 (d, C-8), 26.98 (t, C-9), 33.76 (t, C-10), 28.27 (q, C-11), 27.40 (q, C-12), 51.69 (q, methyl of acetate). - MS (EI, $70 \mathrm{eV}): \mathrm{m} / \mathrm{z}$ $(\%)=360[\mathrm{M}]^{+}(100), 342\left[\mathrm{M}-\mathrm{H}_{2} \mathrm{O}\right]^{+}(12), 327$ $\left[\mathrm{M}-\mathrm{H}_{2} \mathrm{O}-\mathrm{CH}_{3}\right]^{+}(18), 300\left[\mathrm{M}-\mathrm{CH}_{3} \mathrm{COOH}\right]^{+}$ (12), 284 (22), 237 (30), $135\left[\mathrm{C}_{9} \mathrm{H}_{11} \mathrm{O}\right]^{+}$(33), 105 $\left[\mathrm{C}_{9} \mathrm{H}_{11} \mathrm{O}-2 \mathrm{CH}_{3}\right]^{+}(40), 91\left[105-\mathrm{CH}_{2}\right]^{+}$(50). $\mathrm{C}_{21} \mathrm{H}_{28} \mathrm{O}_{5}$ : calcd. 360.1902; found 360.1937 .

\section{Acknowledgement}

The authors thank Prof. Ahmed A. Ahmed for his help, assistance, and his teaching. 
Boriky D., Berrada M., Talbi M., Keravis G., and Rouessac F. (1996), Eudesmanolides from Artemisia herba-alba. Phytochemistry 43, 309-311.

Hu J., Zhu Q., Bai S., and Jia Z. (1996), New eudesmane sesquiterpene and other constituents from Artemisia mongolica. Planta Med. 62, 477-478.

Kim J.-H., Kim H.-K., Jeon S. B., Son K.-H., Kim E. H., Kang S. K., Sung N. D., and Kwon B. M. (2002), New sesquiterpene-monoterpene lactone, artemisolide, isolated from Artemisia argyi. Tetrahedron Lett. 43, 6205-6208.

Kwak J. H., Jang W. Y., Zee O. P., and Lee K. R. (1997), Artekeiskeanin A: a new coumarin-monoterpene ether from Artemisia keiskeana. Planta Med. 63, $474-476$.

Lee S. J., Chung H.-Y., Maier C. G.-A., Wood A. R., Dioxan R. A., and Mabry T. J. (1998), Estrogenic flavonoids from Artemisia vulgaris L. J. Agric. Food Chem. 46, 3325-3329.

Marco J. A. (1989), Sesquiterpene lactones from Artemisia herba-alba subsp. herba-alba. Phytochemistry 28, 3121-3126.

Marco J. A., Sanz J. F., Yuste A., Carda M., and Jakupovic J. (1991), Sesquiterpene lactones from Artemisia barrelieri. Phytochemistry 30, 3661-3668.

Marco J. A., Sanz-Cervera J. F., Ocete G., Carda M., Rodriguez S., and Valles-Xirau J. (1994), New germacranolides and eudesmanolides from North African Artemisia herba-alba. J. Nat. Prod. 57, 939-946.
Mohamed A. E. H., Magdi A. E., Mohamed E. H., Soleiman E. H., Abeer M. E., and Naglaa S. M. (2010), Chemical constituents and biological activities of Artemisia herba-alba. Rec. Nat. Prod. 4, 1-25.

Pathak V. P. and Khanna R. N. (1987), Sesquiterpene lactones from Artemisia maritima. Phytochemistry 26, 2103-2104.

Saadali B., Boriky D., Blaghen M., Vanhaelen M., and Talbi M. (2001), Alkamides from Artemisia dracunculus. Phytochemistry 58, 1083-1086.

Tan R. X., Lu H., Wolfender J. L., Yu T. T., Zheng W. F., Yang L., Gafner S., and Hostettmann K. (1999), Mono- and sesquiterpenes and antifungal constituents from Artemisia species. Planta Med. 65, 64-67.

Tang H. Q., Hu J., Yang L., and Tan R. X. (2000), Terpenoids and flavonoids from Artemisia species. Planta Med. 66, 391-393.

Todorova M. N., Tsankova E. T., Trendafilova A. B., and Gussev C. V. (1996), Sesquiterpene lactones with the uncommon rotundane skeleton from Artemisia pontica L. Phytochemistry 41, 553-556.

Zheng W. F., Tan R. X., Yang L., and Liu Z. L. (1996), Two flavones from Artemisia giraldii and their antimicrobial activity. Planta Med. 62, 160-162. 